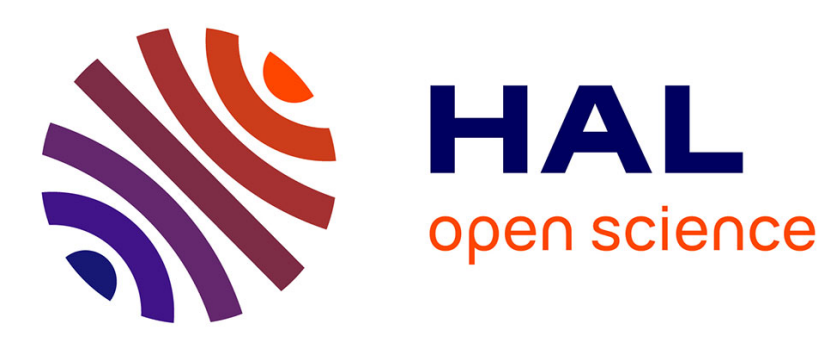

\title{
Hydrodynamic instability and sound amplification over a perforated plate backed by a cavity
}

\author{
Xiwen Dai, Yves Aurégan
}

\section{To cite this version:}

Xiwen Dai, Yves Aurégan. Hydrodynamic instability and sound amplification over a perforated plate backed by a cavity. 25th AIAA/CEAS Aeroacoustics Conference, May 2019, Delft, Netherlands. 10.2514/6.2019-2703 . hal-02337009

\section{HAL Id: hal-02337009 \\ https://hal.science/hal-02337009}

Submitted on 29 Oct 2019

HAL is a multi-disciplinary open access archive for the deposit and dissemination of scientific research documents, whether they are published or not. The documents may come from teaching and research institutions in France or abroad, or from public or private research centers.
L'archive ouverte pluridisciplinaire HAL, est destinée au dépôt et à la diffusion de documents scientifiques de niveau recherche, publiés ou non, émanant des établissements d'enseignement et de recherche français ou étrangers, des laboratoires publics ou privés. 


\title{
Hydrodynamic instability and sound amplification over a perforated plate backed by a cavity
}

\author{
Xiwen Dai* \\ School of Mechanical Engineering, Shanghai Jiao Tong University, Shanghai 200240, China \\ Yves Aurégan ${ }^{\dagger}$ \\ Laboratoire d'Acoustique de l'Université du Mans, Centre National de la Recherche Scientifique (CNRS), \\ Le Mans Université, Avenue Olivier Messiaen, 72085 Le Mans Cedex 9, France
}

\begin{abstract}
The long-wavelength hydrodynamic behaviour over a cavity-backed perforated plate, in a duct with a mean shear flow, is studied numerically using the multimodal method, where the acoustic and hydrodynamic disturbances are calculated from the linearized Euler equations. The flow-acoustic coupling near the perforated plate is first solved hole by hole, and results indicate a well-defined large-scale hydrodynamic wave over the plate, with a wavelength close to the plate length at the peak sound amplification frequency when a plane acoustic wave is introduced from the upstream duct. Since the hydrodynamic wavelength is one order larger than the period of the perforation, the effect of the perforated plate is then described by a homogeneous plate impedance. It is shown that the homogenized approach approximately represents the discrete approach in this problem.
\end{abstract}

\section{Introduction}

T INERS are effective in mitigating noise emissions from aero-engines. The interaction between flow and sound in the vicinity of the lined walls of aero-engine nacelles is complex and, therefore, a precise description of the sound attenuation in a lined duct with flow still attracts lots of attention [1-4]. On the other hand, it is surprising to find that, under certain circumstances, a liner can also act as a sound amplifier [5, 11, 13] or a "singer" [1, 14]. For those phenomena, a type of instability is usually involved in the flow-acoustic coupling.

Flow passing the holes of a perforated plate can give rise to shear layer instability, which causes small-scale instability waves or vortex in each of the holes. A feedback loop consisting of the Kelvin-Helmholtz instability wave and an upstream propagating pulse within each individual small hole can lead to a self-noise tone at a very high frequency [1]. When such instability couples with the acoustic resonant modes of the flow ducts or cavities, strong pure-tone whistling occurs near the frequency of the acoustic resonance [14]. In those cases, the instability waves have wavelengths of the order of the size of the small holes. The same coupling between the shear layer instability in a small opening and acoustic resonant modes inside a cavity can also be found in the self-sustained oscillations in a single cavity [15], 16], a side-branch [17, 18], and a Helmholtz resonator [19, 20].

Hydrodynamic instability with wavelength much larger than the size of the perforations and an associated sound amplification, near the resonance frequency of the liner, have been observed when the liner has a low resistance and the flow velocity is relatively high [5,-8]. The instability is due to the coupling of a hydrodynamic mode in the shear flow with the cavity resonance, and such an instability over the liner can still exist even when the Kelvin-Helmholtz instability of the shear flow over the cavities or holes does not occur. It has also been found that this type instability wave does not scale on any streamwise geometrical dimensions of the liner, i.e. neither the size of the holes nor the total length of the lined wall [13]. A second type large-scale instability over a perforated plate backed by a single cavity, where the instability wavelength is of the order of the total plate length, has also been reported. It was first observed in experiments in water channels that long-wavelength instability along perforated and slotted plates can occur without acoustic or gravity wave resonance [21-23]. The coupling of this large-scale instability with the acoustic [24] and gravity wave [25] resonance of the bounding cavities was also investigated.

The large-scale hydrodynamic instability over a non-local liner, which consists of a cavity covered by a same-length perforated plate, with a wavelength close to the plate length is considered in this paper. The problem is sketched in Fig. 1. where the non-local liner is attached to a duct containing a mean shear flow. Both discrete approach where

\footnotetext{
*Associate professor, School of Mechanical Engineering, xiwen.dai@sjtu.edu.cn.

†'Research director, Laboratoire d'Acoustique de 1'Université du Maine, yves.auregan@univ-lemans.fr.
} 


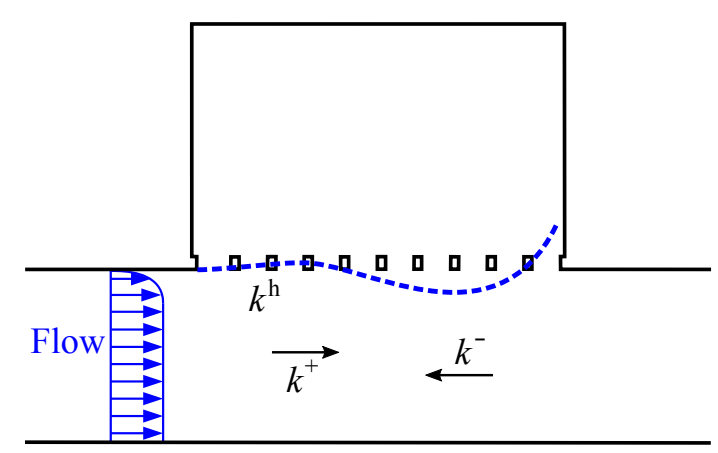

Fig. 1 Sketch of the flow-acoustic coupling over a perforated plate baked by a cavity
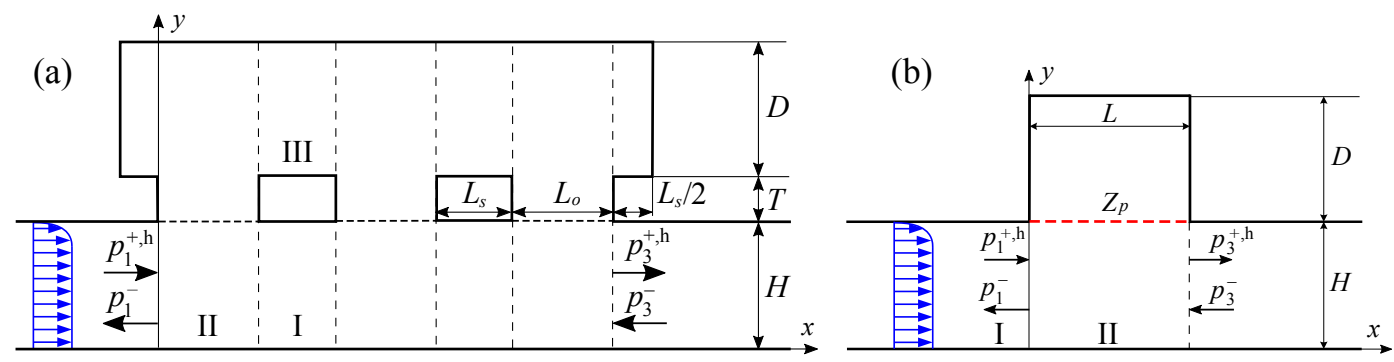

Fig. 2 Sketches of the hole-by-hole approach (a) and the homogenized approach (b) of modal scattering.

the flow-acoustic coupling at the perforated plate is described hole-by-hole and homogenized approach in which the perforated plate is represented by a homogeneous impedance are used to model this large-scale hydrodynamic behaviour.

\section{Model}

Calculation of the modal scattering of a non-local liner attached to a flow duct containing a mean shear flow is sketched in Fig. 2. To solve this problem of linear propagation in a shear flow, the multimodal method is used [26, 27], where the disturbances in the ducts are expressed as a linear superposition of acoustic and hydrodynamic tranverse modes. For details of the modal scattering calculation in a duct-cavity system with a shear flow, the reader is also referred to [12, 13].

In the discrete approach, as sketched in Fig. 2(a), the configuration is divided into zones of three types denoted by I, II, and III. In each zone, the modal profiles and wavenumbers of transverse modes are solved numerically. In zones I and II, duct modes include acoustic modes propagating or decaying in the $\pm x$ directions and hydrodynamic modes travelling in the $+x$ direction with the mean flow. Zone III does not have mean flow, so only acoustic modes are solved. A resistance $R$ denoted by the horizontal dashed lines in Fig. 2(a) is introduced at the entrenance of each hole in the plate to mimic the resistance without flow due to thermo-viscous effects. The transverse modes in each zone are then matched using the continuity of pressure $p$, velocity $v$, and $\partial p / \partial x$ at the interfaces between zones, and $\partial p / \partial x=0$ on the vertical walls inside the small holes and the backing cavity. The continuity and wall conditions can be put in the form of a large matrix that links incoming waves to out-going waves and to all the internal variables in the non-local liner. From this large matrix, the scattering matrix of the liner is obtained.

In a homogenized approach, the perforated plate is described by its acoustic impedance $Z_{p}$ denoted by the red dashed lines in Fig. 2 (b), which leads to a pressure jump at $y=1$ for $0<x<L$. Duct modes are solved in each zone, and the modal matching at the interfaces between the zones gives the scattering matrix. 


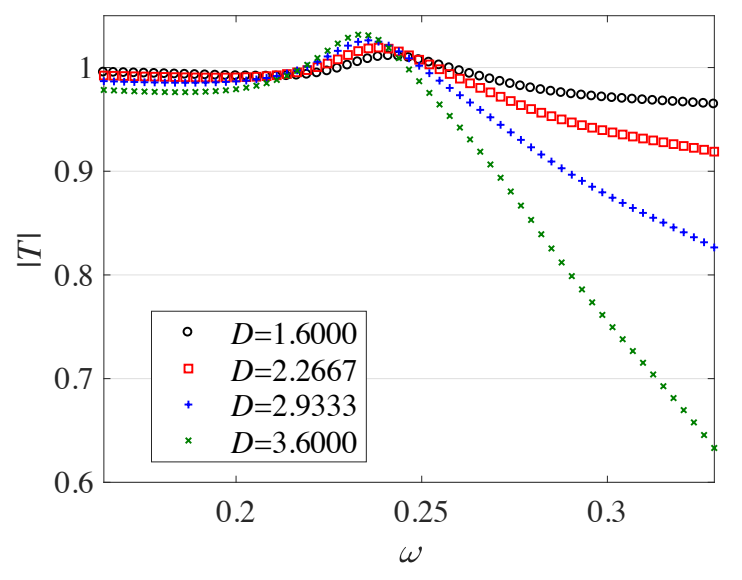

Fig. 3 Transmission coefficients for a plane acoustic wave passing through the liner in the flow direction, calculated with the hole-by-hole description of the perforated plate.

\section{Results}

The geometrical parameters are: $H^{*}=15 \mathrm{~mm}, T^{*}=1 \mathrm{~mm}, L_{o}^{*}=1 \mathrm{~mm}$, and $L_{s}^{*}=0.2 \mathrm{~mm}$. The perforated plates contains 10 holes, thus the length of the plate and the backing cavity is $L^{*}=12 \mathrm{~mm}$. The Mach number averaged over the cross-section is $M_{0}=0.1$ and the velocity profile is given by a simple polynomial law with a unity average value, $f=\left(1-y^{m}\right)(m+1) / m$, where the parameter $m=10$ is used. At the entrance of each hole a thin sheet with a normalized resistance $R=0.016$ has been added. The number of the discrete points in the duct is 300 , so 900 transverse modes are solved from in the duct. Transmission coefficient for a plane acoustic wave passing through the cavity segment in the flow direction is presented in figure 3 for four different cavity depths: $D^{*}=24 \mathrm{~mm}, 34 \mathrm{~mm}, 44 \mathrm{~mm}$ and $54 \mathrm{~mm}$. It is observed that sound can be amplified by the liner at certain frequencies.

The fields of the pressure $p$ and transverse velocity $v$ are shown in Fig. 4(a,b) at the peak sound amplification frequency. A convectively growing wave appears over the perforated plate. The amplitude and phase of $v$ approximately remain the same across the plate, as shown in Fig. 4.c,d), which are in agreement with experimental results of [22]. It can also be found in Fig. 4(c) that the growth always happens in the small holes, which can be understood as a result of the Kelvin-Helmholtz instability of the shear flow [28] in the holes. However, the observed wavelength is close to the length of the perforated plate $L$ rather than the individual holes $L_{o}$, as shown by the fields and also by the phase angle in Fig. 4(d). In other words, the Strouhal number of the sound amplification scales on the length of the perforated plate rather than the holes $\left(S r=\omega L / U_{0}\right)$. This means that it is difficult to explain the large-scale hydrodynamic wave and the related sound amplification by only examining the instabilities in the individual holes. Moreover, the long wavelength, compared to the period of the perforated plate (for the present case $\lambda_{h y} /\left(L_{o}+L_{S}\right) \approx 10$ ), suggests that a homogenized description of the perforated plate can be used in this problem.

To understand the large-scale convectively growing wave over the perforated plate, the simplified model based on the plate impedance will be utilized in the following analysis. Also, since the goal is not to describe every detail of the flow and acoustics, but rather to obtain a better understanding of the phenomenon, the classic and simple plate impedance model of [29] is used to describe the perforated plate with grazing flow, $Z_{p}=\left(R+R_{f}\right) / \sigma+\mathrm{i} \omega(T+\delta) / \sigma$ where, for a low sound pressure level: $R_{f}=0.3\left(1-\sigma^{2}\right) M_{0}$ and $\delta=0.85 L_{o}(1-0.7 \sqrt{\sigma})\left(1+305 M_{0}^{3}\right)^{-1}$, and $\sigma$ is the open area ratio of the perforated plate $\sigma=L_{o} /\left(L_{o}+L_{s}\right)$. The results of the transmission coefficient for a plane acoustic wave passing through the liner in the flow direction are shown in Fig. 5. Compared to results in Fig. 3, the amplitudes are over predicted, which could be due to that the effect of the flow on the plate resistance is underestimated. However, the amplification frequencies and the trend of the peak amplification frequency and amplitude with the increasing cavity depth are well captured by the simplified model.

Iso-colour plots of the real part of $p$ and $v$ at the peak amplification frequency shown in Fig. 5 for $D=2.933$ are presented in Fig. 6(a,b). A large-scale convectively growing hydrodynamic wave with wavelength close to the length of the liner can be seen. Comparison of the fields in Figs. 4 and 6 , respectively from the discrete and homogenized models for the perforated plate, shows a qualitative agreement. The variation of the transverse velocity along the perforated 

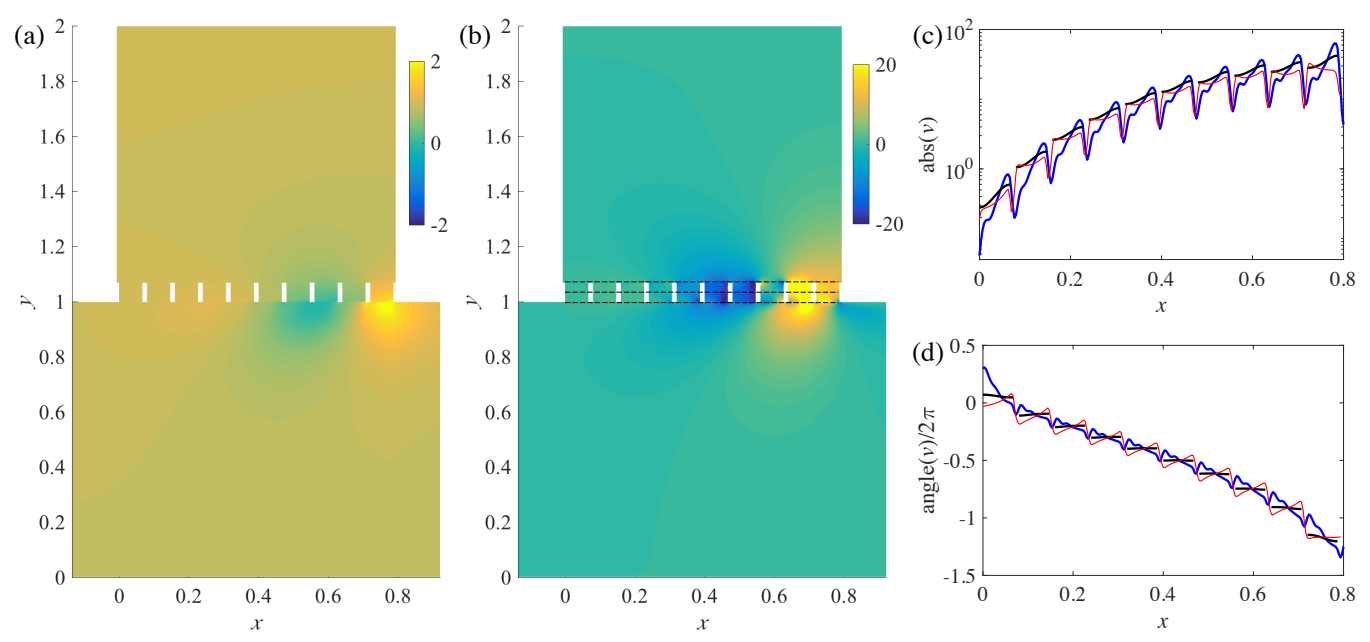

Fig. 4 Iso-colour plots of the real part of $p$ (a) and $v$ (b) for a plane sound wave incidence at the peak amplification shown in Fig. 3. In the calculation, $D=2.9333(44 \mathrm{~mm})$ and $\omega=0.2356(860 \mathrm{~Hz})$. Note that the fields are only plotted for the duct, the perforated plate, and part of the backing cavity. (c) and (d) are the amplitude and phase angle of $v$ along the perforated plate at $y$ positions (blue thick lines: just below the plate; black segmented thick lines: in the middle of the holes of the plate; red thin lines: just above the plate), denoted by the dashed-lines in (b).

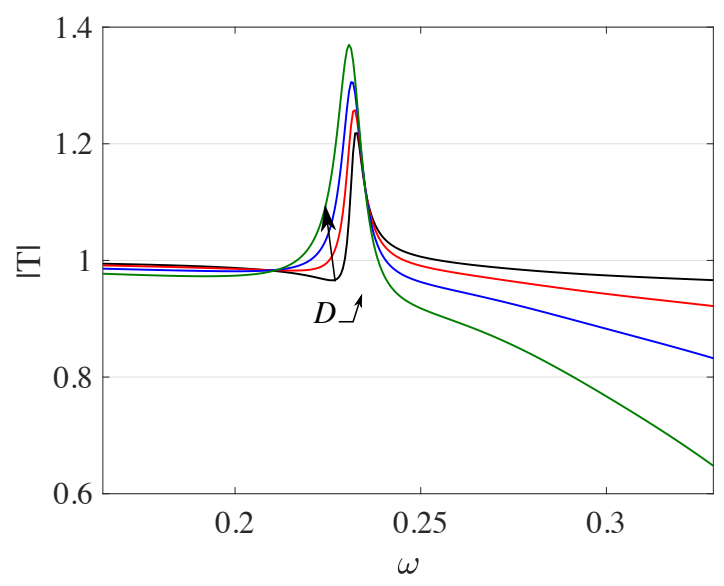

Fig. 5 Transmission coefficients for a plane acoustic wave passing through the liner in the flow direction, calculated with the impedance description of the perforated plate. The depths of the backing cavity are the same as in figure 3 . 

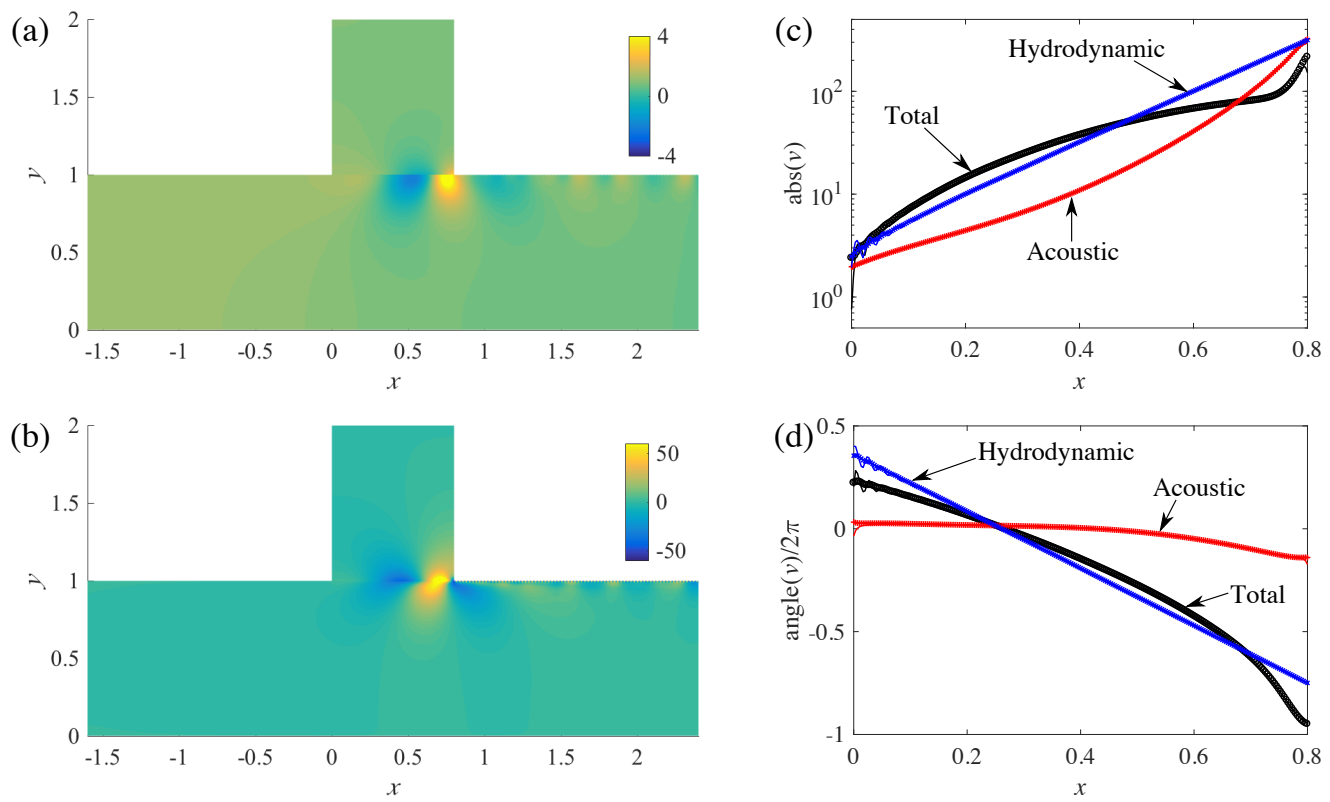

Fig. 6 Iso-colour plots of the real part of $p$ (a) and $v$ (b) for a plane sound wave incidence at the peak amplification shown in Fig. 5. In the calculation, $D=2.9333(44 \mathrm{~mm})$ and $\omega=0.2315(845 \mathrm{~Hz})$. Note that the fields are only plotted for the duct and part of the backing cavity. (c) and (d) are the amplitude and phase angle of $v$ along the cavity mouth (symbols: just below $y=1$; lines: just above $y=1$ ).

plate are given in Fig. 6(c,d). The exponential growth along the plate in the amplitude of the hydrodynamic part of the velocity indicates that the unstable hydrodynamic mode of the shear flow is excited at the upstream end of the plate. The slope of the phase angle of the hydrodynamic velocity is constant, which means that such unstable mode is convected downstream at a constant velocity. The acoustic velocity also has a growing amplitude with increasing $x$ along the plate, especially near the downstream end, and the phase angle only changes where $x>0.5$ but almost keeps unvarying in the range $0<x<0.5$. These suggest that the acoustic waves are excited near the downstream edge, and they are mainly the high-order modes. Thus, sound amplification comes from the excited unstable hydrodynamic mode that scatters sound waves near the downstream edge.

\section{Conclusion}

The long-wavelength hydrodynamic instability over a non-local liner, which consists of a cavity covered by a same-length perforated plate, in a flow duct is studied by a two-dimensional (2D) linear analysis. The mean shear flow in the duct is assumed unchanged in the streamwise direction and the acoustic and hydrodynamic disturbances are described by the linearized Euler equations with an artificial damping at the entrance of each hole in the plate. The results of the hole-by-hole approach show that the present simple model can describe the appearance of the large-scale instability over the non-local liner. The homogenized approach approximately represents the hole-by-hole approach in describing the large-scale instability. However, the plate impedance model used can lead to inaccuracy in describing wave propagation, especially when the flow velocity is high [12]. For this reason, only a qualitative comparison can be made between the discrete and homogenized models, and a quantitative agreement requires a more precise homogenization of a perforated plate with flow.

\section{Acknowledgments}

X.D. gratefully acknowledges support from the National Natural Science Foundation of China no. 51876120; Y.A. gratefully acknowledges support from the ANR international project FlowMatAc no. ANR-15-CE22-0016-01. 


\section{References}

[1] Tam, C. K.W., Pastouchenko, N. N., Jones, M. G. \& Watson, W. R. 2014 Experimental validation of numerical simulations for an acoustic liner in grazing flow: self-noise and added drag. J. Sound Vib. 333, 2831-2854.

[2] Zhang, Q. \& Bodony, D. J. 2016 Numerical investigation of a honeycomb liner grazed by laminar and turbulent boundary layers. J. Fluid Mech. 792, 936-980.

[3] Khamis, D. \& Brambley, E.J. 2016 Acoustic boundary conditions at an impedance lining in inviscid shear flow. J. Fluid Mech. 796, 386-416.

[4] Khamis, D. \& Brambley, E.J. 2017 Viscous effects on the acoustics and stability of a shear layer over an impedance wall. $J$. Fluid Mech. 810, 489-534.

[5] Brandes, M. and Ronneberger D., "Sound amplification in flow ducts lined with a periodic sequence of resonators," AIAA peper 95-126, 1995, pp. 893-901.

[6] Ronneberger, D. and Jüschke, M. 2007 Sound absorption, sound amplification, and flow control in ducts with compliant walls, In Oscillations, Waves and Interactions (Ed. T. Kurz, U. Parlitz, and U. Kaatze) pp 73-106, Universitätsverlag Göttingen.

[7] Aurégan, Y. and Leroux, M., "Experimental evidence of an instability along an impedance wall with flow," J. Sound Vib., Vol. 317, 2008, pp. 432-439.

[8] Marx, D., Aurégan, Y., Bailliet, H., \& Valière, J.-C. 2010 PIV and LDV evidence of hydrodynamic instability over a liner in a duct with flow. J. Sound Vib. 329, 3798-3812.

[9] Marx, D. \& Aurégan, Y. 2013 Effect of turbulent eddy viscosity on the unstable surface mode above an acoustic liner. J. Sound Vib. 332, 3803-3820.

[10] Aurégan, Y. and Singh D.K., "Experimental observation of a hydrodynamic mode in a flow duct with a porous material," $J$. Acoust. Soc. Am., Vol. 136, 2014, pp. 567-572.

[11] Alomar, A. and Aurégan, Y., "Particle image velocimetry measurement of an instability wave over a porous wall in a duct with flow", J. Sound Vib., Vol. 386, 2017, pp. 208-224.

[12] Dai, X. \& Aurégan, Y. 2016 Acoustic of a perforated liner with grazing flow: Floquet-Bloch periodical approach versus impedance continuous approach. J. Acoust. Soc. Am. 140, 2047-2055.

[13] Dai, X. \& Aurégan, Y. 2018 A cavity-by-cavity description of the aeroacoustic instability over a liner with a grazing flow. $J$. Fluid Mech. 825, 126-145.

[14] Tsui, C.Y. \& Flandro, G.A. 1977 Self-induced sound generation by flow over perforated duct liners. J. Sound Vib. 50, 315-331.

[15] East, L. F. 1966 Aerodynamically induced resonance in rectangular cavities. J. Sound Vib. 3, 277-287.

[16] Yamouni, S., Sipp, D. \& Jacquin, L. 2013 Interaction between feedback aeroacoustic and acoustic resonance mechanisms in a cavity flow: a global stability analysis. J. Fluid Mech. 717, 134-165.

[17] Bruggeman, J. C., Hirschberg, A., van Dongen, M. E. H. \& Wijnands, A. P. J. 1991 Self-sustained aero-acoustic pulsations in gas transport systems: Experimental study of the influence of closed side branches. J. Sound Vib. 151, 371-393.

[18] Ziada, S. \& Shine, S. 1999 Strouhal numbers of flow-excited acoustic resonance of closed side branches. J. Fluid. Struct. 13, $127-142$.

[19] Ma, R., Slaboch, P. E., \& Morris, S. C. 2009 Fluid mechanics of the flow-excited Helmholtz resonator. J. Fluid Mech. 623, $1-26$.

[20] Dai, X., Jing, X. \& Sun, X. 2015 Flow-excited acoustic resonance of a Helmholtz resonator: Discrete vortex model compared to experiments. Phys. Fluids 27, 057102.

[21] Celik, E. \& Rockwell, D. 2002 Shear layer oscillation along a perforated surface: a self-excited large-scale instability. Phys. Fluids 14, 4444-4447.

[22] Celik, E. \& Rockwell, D. 2004 Coupled oscillations of flow along a perforated plate. Phys. Fluids 16, 1714-1724.

[23] Sever, C. \& Rockwell, D. 2005 Oscillations of shear flow along a slotted plate: small- and large-scale structures. J. Fluid Mech. 530, 213-222. 
[24] Zoccola, P. J. 2004 Effect of opening obstructions on the flow-excited response of a Helmholtz resonator. J. Fluid. Struct. 19, 1005-1025.

[25] Ekmekci, A. \& Rockwell, D. 2007 Oscillation of shallow flow past a cavity: Resonant coupling with a gravity wave. J. Fluid. Struct. 23, 809-838.

[26] Kooijman,G., Testud, P., Aurégan, Y. \& Hirschberg, A. 2008 Multimodal method for scattering of sound at a sudden area expansion in a duct with subsonic flow. J. Sound Vib. 310, 902-922.

[27] Kooijman, G., Hirschberg, A. \& Aurégan, Y. 2010 Influence of mean flow profile and geometrical ratios on scattering of sound at a sudden area expansion in a duct. J. Sound Vib. 329, 607-626.

[28] Schmid, P.J. \& Henningson, D.S. 2000 Stability and Transition in Shear Flows, Springer.

[29] Guess, A. W. 1975 Calculation of perforated plate liner parameters from specified acoustic resistance and reactance. J. Sound Vib. 40, 119-137. 